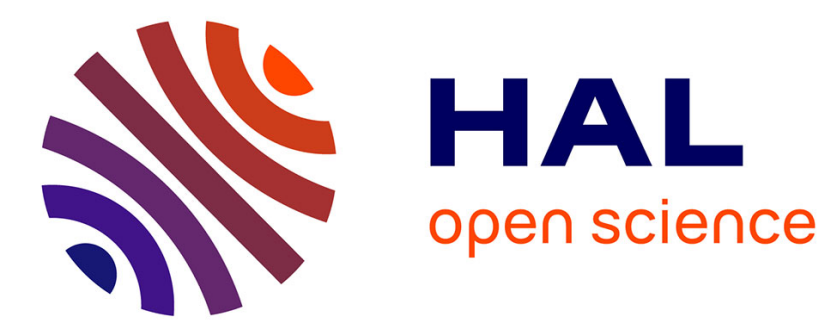

\title{
Control of sound radiation and transmission by a piezoelectric plate with an optimized resistive electrode
}

Giuseppe Rosi, Joël Pouget, Francesco Dell'Isola

\section{To cite this version:}

Giuseppe Rosi, Joël Pouget, Francesco Dell'Isola. Control of sound radiation and transmission by a piezoelectric plate with an optimized resistive electrode. 2010. hal-00507877

\section{HAL Id: hal-00507877 \\ https://hal.science/hal-00507877}

Preprint submitted on 2 Aug 2010

HAL is a multi-disciplinary open access archive for the deposit and dissemination of scientific research documents, whether they are published or not. The documents may come from teaching and research institutions in France or abroad, or from public or private research centers.
L'archive ouverte pluridisciplinaire HAL, est destinée au dépôt et à la diffusion de documents scientifiques de niveau recherche, publiés ou non, émanant des établissements d'enseignement et de recherche français ou étrangers, des laboratoires publics ou privés. 


\title{
Control of sound radiation and transmission by a piezoelectric plate with an optimized resistive electrode
}

\author{
Giuseppe Rosi ${ }^{\mathrm{a}, \mathrm{b}, \mathrm{c}}$, Joël Pouget ${ }^{\mathrm{b}}$, Francesco dell'Isola ${ }^{\mathrm{a}, \mathrm{c}}$ \\ ${ }^{a}$ Dipartimento di Ingengeria Strutturale e Geotecnica, Sapienza Università di Roma, Via Eudossiana \\ 18, 00184, Rome, Italy \\ ${ }^{b}$ Institut Jean Le Rond d'Alembert (CNRS - UMR 7190), Université Pierre et Marie Curie-Paris 6, 4 \\ Place Jussieu, 75252, Paris, France \\ ${ }^{c}$ Laboratorio Strutture e Materiali Intelligenti c/o Fondazione Tullio Levi Civita, Via S. Pasquale \\ s.n.c. Cisterna di Latina (Latina) Italia
}

\begin{abstract}
The aim of this work is the application of the piezoelectric effect to the reduction of the sound radiation transmitted through and radiated by an aluminium panel immersed in a light fluid. An innovative Piezoelectric Resistive Electrode (PRE) plate will be introduced here. This structure consists of an aluminium plate entirely covered by two piezoelectric layers with a controlled resistivity electrode bonded on each free piezoelectric surface. This homogeneous system is optimized for all frequencies and performs a good trade off between good performances and easy implementation. The results will be presented by considering standard acoustic parameters, such as the Sound Reduction Index, far field sound pressure and radiated sound power at plate level. It is shown how this novel smart structure can reduce the sound transmission between two fluid media. To this end, we consider an infinite plate separating two half spaces filled with two identical light fluids, and we analyse how a sound wave is transmitted through the plate. The power of the sound and the far field pressure radiated by a finite structure due to a mechanical input, e.g. a point force, will also be considered. A detailed description of the dynamic and acoustic behaviour of the structure is presented. The performances of the PRE plate are compared with those of a standard viscoelastic damping strategy and of other passive piezoelectric smart structures.
\end{abstract}

Email address: giuseppe.rosi@uniroma1.it (Giuseppe Rosi) 


\section{Introduction}

One of the main aspects which contributes to the valuation of the overall quality of a certain vehicle, such as a car, a ship or an aircraft, is the noise propagating into the passenger compartment due to the vibrations of the structure or to an external source. The study of structural vibrations, structure-borne sound and air-borne sound is a key topic in the automotive, naval and aeronautic industry. In order to adequately model a mechanical component, like a car door or an aeronautic structural panel with two dimensions prevailing over the other, plane structures will be considered.

The use of piezoelectric elements for vibration control is widely diffused in the literature. Connecting a mechanical and an electrical system creates an energy flow, from the mechanical form to the electrical form and vice versa. Subtracting mechanical energy and dissipating it into a non-conservative electric circuit is a means to damp mechanical vibrations. The control technique is then related to the optimization of the energy flow and the non-conservative part of the circuit. There exist two main branches of vibration control: active and passive control. Even though each of these approaches has its advantages and drawbacks, this study is focused on passive control. Examples of active control systems can be found in (Carneal and Fuller, 2004; Lee and Chen, 1999b,a; Strassberger and Waller, 2000; Gardonio and Elliott, 2005; Gardonio et al., 2004). The choice of passive control law was made because a passive control system cannot supply energy to the structure, and so it cannot affect the stability of the system itself. Another reason is that a passive circuit does not need, in principle, any external power supply. When considering passive control laws two main strategies are possible: localized control and distributed. The first approach uses a control system with optimally placed piezoelectric patches (Halim and Moheimani, 2003), connected to a circuit operating in selected frequency windows. With respect to the distributed controller this scheme uses a smaller number of piezoelectric elements and, usually, a simpler circuit. Examples can be found in (Carneal and Fuller, 2004; Behrens et al., 2003; Lee and Chen, 1999b; Zhang et al., 2004; Ozer and Royston, 2003). However, it can introduce a performance trade-off in terms of amount of vibrational energy transferred to the circuit.

A different approach for solving the same problem considers a distributed piezoelectric network connected to a virtual passive modular circuit, optimized for harvesting me- 


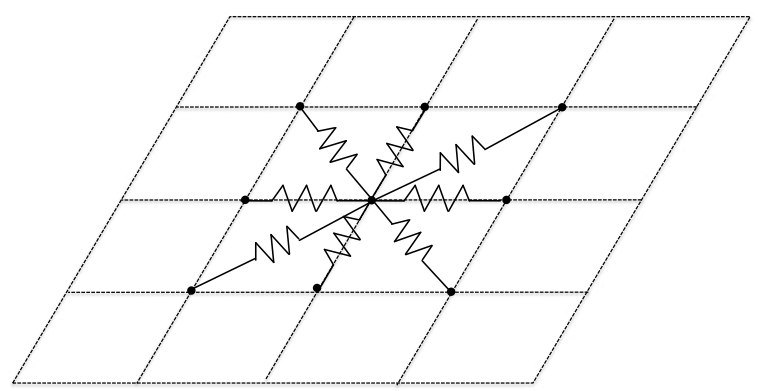

Figure 1: Optimal purely resistive interconnection for discrete distributed systems

chanical energy of the plate and dissipating it into the circuit. The uniform spatial distribution of sensors and actuators permits to have, with optimized electrical parameters, the duality between the electrical circuit and the mechanical structure. This duality traduces in the superposition of the electric and mechanic eigenfrequencies and in an optimal energy flow. This passive technique assures stability and optimized broadband control. Applications of this technique can be found in (Maurini, 2005; dell'Isola et al., 2003; Alessandroni et al., 2004, 2002).

Finding the solution of the acoustic problem implies difficult calculations and sometimes an analytical solution of the considered problem does non even exist. Following (Wallace, 1972; Elliott and Johnson, 1993; Borgiotti and Jones, 1994; Bai and Tsao, 2002) the elementary radiator decomposition and the concept of radiation spatial filters will be used in order to reduce the computational cost of the analytical treating and to make the synthesis of the controller easier. With the study of the dispersion relations of the waves, propagating through the fluid and the structure, it is possible to obtain optimal parameters for the components and for the acoustic purposes. This is a crucial step for the optimization of the control strategy and for developing an ad-hoc controller. When considering discrete distributed systems, it is known from the literature (Maurini et al., 2004) that there exist several optimal configurations for the electric circuit for optimizing the vibration damping through piezoelectric coupling. Being the piezoelectric transducers modelled as a capacitor in parallel with a current generator, inductors permit to have resonances in the electric circuit, but as the optimal value to assure the tuning is often too big for a passive realization, or even negative, they have to be simulated with active circuits. That is why in this work we will introduce a purely resistive networks. The 


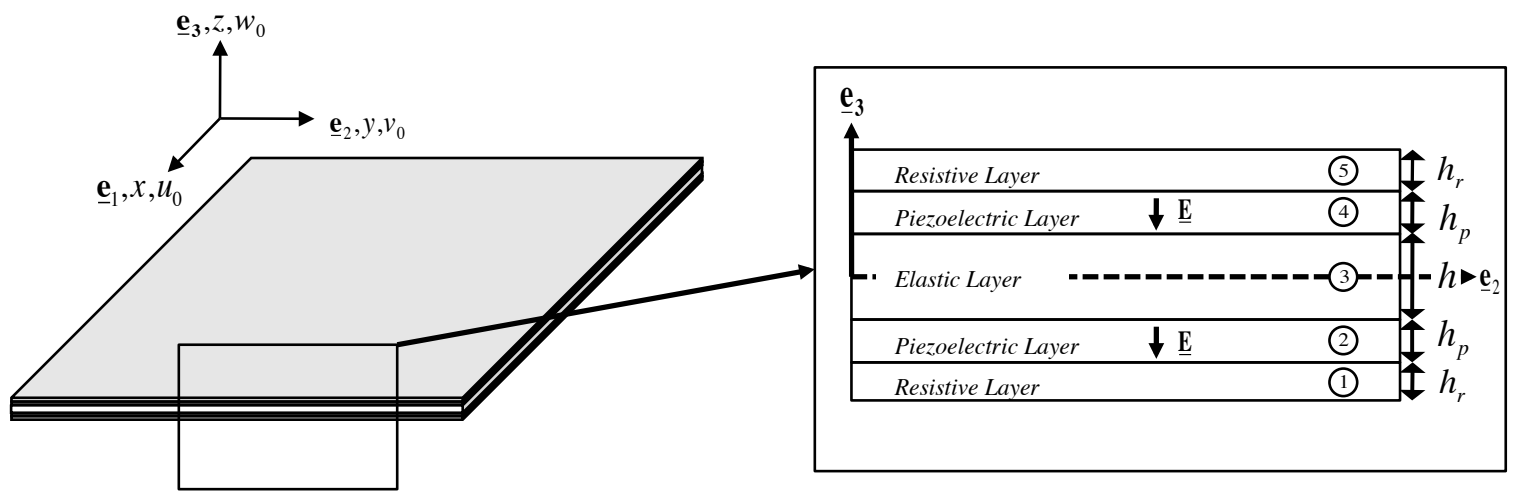

Figure 2: Geometry of the PRE plate

advantage in using distributive networks is that the spatial distribution of the electric elements makes the circuit able to adapt, in our case, the energy dissipation. Usually this optimal network is synthesized interconnecting a matrix of piezoelectric actuators with a passive network of resistors (figure 1). For the dimensions of the transducers smaller than the wavelength of the vibration propagating into the structure, this discrete system can be homogenized. Throughout the paper we will show how this discrete network can be replaced with a resistive electrode bonded on a unique piezoelectric patch. A schematic description of the system is in figure 2 .

In the first part of the paper the equations of motion for the piezoelectric plate will be derived, starting from kinematics of each layer, using the principle of virtual work and introducing the constitutive equations. The second part will be devoted to the optimization of the parameters in order to maximize the performances of the structure. The application of this novel system to the sound radiation and transmission control will be the object of the final part of this paper.

\section{The Model of Piezoelectric Resistive Electrode (PRE) Plate}

The aim of this section is to derive the model of the PRE plate using a variational principle. We will start considering the geometry and the kinematics of the structure, then we will formulate the virtual work principle. Next we will derive the constitutive equations for the PRE plate and, finally, obtain the equations of motion. 


\subsection{Geometry}

We consider a Kirchhoff-Love (K-L) plate with piezoelectric layers bonded on the two sides. The choice of considering a K-L plate theory instead of the more general Reissner-Mindlin (R-M) plate theory is motivated by to the fact that the approximations introduced the reduced K-L plate do not affect our results considerably. This because the plate is considerably much thinner than the considered wavelengths. The free surface of each piezoelectric layer is covered by a resistive layer, the other electrode is the aluminium plate itself, figure 2 . We assume that the plate is composed of a homogeneous and transversely isotropic material. Piezoelectric elements are polarized along the thickness. We identify the plate by its reference shape $B$. Let $S$ be a flat surface, $\underline{e}_{3}$ the corresponding normal unit vector, $\underline{r}$ a generic vector in the plane of the plate and $I$ an interval corresponding to the plate thickness.

We assume that

$$
B=\left\{\underline{r}+z \underline{e}_{3}, \underline{r} \in S, z \in I\right\} .
$$

Since the plate, figure 2 , is composed of five layers we have that the interval $I$ is naturally partitioned as $I=\bigcup_{i} I_{i}$. Consequently $B$ is decomposed as $B=\bigcup_{i} B_{i}$, where $B_{3}$ is the elastic layer, $B_{2}$ and $B_{4}$ are piezoelectric layers, whilst $B_{1}$ and $B_{5}$ are resistive layers.

The following sets of indexes are considered:

$$
i=\{1,2,3,4,5\}, \quad l=\{2,4\}, \quad r=\{1,5\} .
$$

The value of intervals is (see figure 2 for the layer thickness definitions)

$$
\begin{array}{ll}
I_{1}=\left[-\frac{h}{2}-h_{p}-h_{r},-\frac{h}{2}-h_{p}\right], & I_{2}=\left[-\frac{h}{2}-h_{p},-\frac{h}{2}\right], \\
I_{3}=\left[-\frac{h}{2}, \frac{h}{2}\right], & I_{4}=\left[\frac{h}{2}, \frac{h}{2}+h_{p}\right], \\
I_{5}=\left[\frac{h}{2}+h_{p}, \frac{h}{2}+h_{p}+h_{r}\right] . &
\end{array}
$$

The boundary of $B$ can be decomposed in the lateral boundary $\partial S \times I$ and in the upper and lower faces $S \times I^{+}$and $S \times I^{-}$. Where $I^{+}=\max \left[I_{5}\right]$ and $I^{-}=\min \left[I_{1}\right]$.

\subsection{Kinematics}

In this section the kinematic restrictions for each kind of layer will be introduced. 


\subsubsection{Kinematic restrictions for the elastic layer}

According to the K-L plate theory, the following assumption for the displacement field is considered:

$$
\underline{u}(x, y, z, t)=\underline{u}_{0}(x, y, t)+w_{0}(x, y, t) \underline{e}_{3}-z \nabla w_{0}(x, y, t)
$$

where $w_{0}$ is the transverse displacement,

$$
\underline{u}_{0}(x, y, t)=u_{0}(x, y, t) \underline{e}_{1}+v_{0}(x, y, t) \underline{e}_{2}
$$

is the displacement of the middle plane and the differential operator is defined as follows

$$
\nabla=\frac{\partial}{\partial x} \underline{e}_{1}+\frac{\partial}{\partial x} \underline{e}_{2}
$$

The corresponding strain field is

$$
\mathbf{S}\left(\underline{u}_{0}, w_{0}\right)=\mathbf{S}_{0}(\underline{u})-z \mathbf{S}_{1}\left(w_{0}\right),
$$

where

$$
\mathbf{S}_{0}\left(\underline{u}_{0}\right)=\operatorname{sym}\left(\nabla \underline{u}_{0}\right), \quad \mathbf{S}_{1}\left(w_{0}\right)=\operatorname{sym}\left(\nabla^{2} w_{0}\right) .
$$

\subsubsection{Kinematic restrictions for the piezoelectric layers}

In each piezoelectric layer the assumptions for the electric potential $\varphi^{(l)}(x, y, z, t)$ and electric field $\underline{E}^{(l)}(x, y, z, t)$ are

$$
\begin{gathered}
\varphi^{(l)}(x, y, z, t)=\varphi_{0}^{(l)}(x, y, t)+z \varphi_{1}^{(l)}(x, y, t), \\
\underline{E}^{(l)}(x, y, t)=-\operatorname{grad}\left(\varphi^{(l)}\right)=-\frac{d \varphi^{(l)}}{d z} \underline{e}_{3} .
\end{gathered}
$$

These conditions imply that, being $V^{(l)}$ the voltage at the interfaces between the piezoelectric and resistive layer, and being $\psi^{(l)}=\int V^{(l)} d t$ the flux linkage at the interfaces, the electric field is constant along the thickness and its value is

$$
\underline{E}^{(l)} \cdot \underline{e}_{\mathbf{3}}=E_{3}^{(l)}=\varpi^{(l)} \frac{V^{(l)}}{h_{p}}=\varpi^{(l)} \frac{\dot{\psi}^{(l)}}{h_{p}},
$$

where the value of $\varpi^{(l)}$ depends on the polarization of the $l-t h$ layer. As shown in figure 2 it is $\varpi^{(2)}=-1$ and $\varpi^{(4)}=1$. A schematic diagram of voltage distribution along the thickness is displayed in figure 3 . 


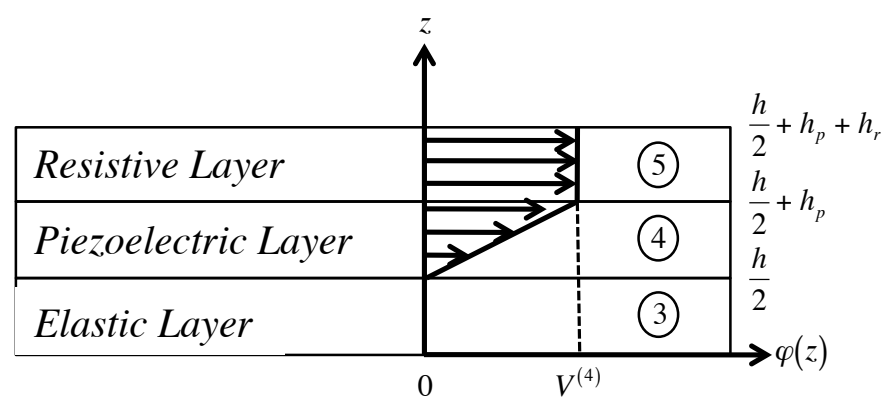

Figure 3: Voltage distribution along the thickness.

\subsubsection{Kinematic restrictions for the resistive layers}

For the resistive layer we suppose that the value of the voltage $V^{(l)}$ is constant along the thickness, so that $\partial V^{(r)} / \partial z=0$. The kinematic at the interfaces between the elastic and piezoelectric layer are

$$
V^{(1)}=V^{(2)}, \quad V^{(4)}=V^{(5)},
$$

and then

$$
\dot{\psi}^{(1)}=\dot{\psi}^{(2)}, \quad \dot{\psi}^{(4)}=\dot{\psi}^{(5)},
$$

where $\dot{\square}$ denotes a partial time derivative.

\subsection{Principle of virtual work and balance equations}

According to the kinematic conditions introduced in section 2.2, in this section, a variational principle will be applied to the structure, in order to obtain balance equations and well-posed boundary conditions.

\subsubsection{Internal work}

In order to apply principle of virtual work a suitable expression for the internal energy must be found. Introducing $\mathbf{T}, \underline{D}$ and $\underline{J}$, as the stress tensor, the electric displacement vector and the current density vector, and defining by $\widetilde{\square}$ a virtual quantity, we can write the internal work $L_{i n t}$ as

$$
L_{i n t}=\underbrace{\delta E^{m}\left(\mathbf{T}\left(\underline{u}_{0}, w_{0}\right), \mathbf{S}\left(\underline{\tilde{u}}_{0}, \tilde{w}_{0}\right)\right)}_{\text {Elastic energy }}+\underbrace{\delta E^{e}(\underline{D}(\psi), \underline{\tilde{E}}(\tilde{\psi}))}_{\text {Electric energy }}+\underbrace{L^{d}(\underline{J}(\psi), \nabla \tilde{\psi})}_{\text {Dissipated energy }} .
$$


In equation (4) there are two conservative terms, the variation of elastic energy $\delta E^{m}$ and the variation of the electric energy $\delta E^{e}$, and a non-conservative term $L^{d}$ of the energy dissipated into each resistive layer by Joule heat. For the kinematic conditions introduced in each layer we have

$$
\begin{gathered}
\delta E^{m}=\int_{S} \int_{I} \mathbf{T}_{\wp}: \operatorname{sym}\left[\nabla \underline{\tilde{u}}_{0}-z \nabla^{2} \tilde{w}_{0}\right], \\
\delta E^{e}=\int_{S} \int_{I} \frac{\underline{D} \cdot \underline{e}_{3}}{h_{p}} \tilde{V}, \\
L^{d}=\int_{S}\left(\sum_{r} \underline{J}^{(r)} \cdot \nabla \tilde{\psi}\right) h_{r},
\end{gathered}
$$

where $\mathbf{T}_{\wp}=\mathbf{T}\left(\mathbf{I}-\underline{e}_{3} \otimes \underline{e}_{3}\right)$ is the projection of $\mathbf{T}$ on the plane perpendicular to $\underline{e}_{3}$. Introducing the terms

$$
\mathbf{N}=\int_{I} \mathbf{T}_{\wp}, \quad \mathbf{M}=\int_{I}-z \mathbf{T}_{\wp}, \quad q=\int_{I} \frac{\underline{D} \cdot \underline{e}_{3}}{h_{p}},
$$

we can write the final form for the internal energy

$$
L_{i n t}=\underbrace{\int_{S} \mathbf{N}: \operatorname{sym}\left(\nabla \tilde{u}_{0}\right)}_{(a)}+\underbrace{\int_{S} \mathbf{M}: \nabla^{2} \tilde{w}_{0}}_{(b)}+\underbrace{\int_{S} q \tilde{V}}_{(c)}+\underbrace{\int_{S} \sum_{r} \underline{J}^{(r)} \cdot \nabla \tilde{\psi} h_{r}}_{(d)} .
$$

where (a) is the virtual work of the forces acting on the plane $\left(\mathbf{I}-\underline{e}_{3} \otimes \underline{e}_{3}\right)$, (b) that of the bending moments, (c) that of the electric forces and (d) is the non conservative energy dissipated within each resistive layer.

\subsubsection{External work}

In order to apply the principle of virtual work we now have to define the work done by the external forces and electric entities on the virtual displacements. Introducing $\underline{b}$ and $f$ as the body and surface forces acting on $B$ including the acceleration forces we can write for the virtual work of a generic virtual displacement

$$
L_{e x t}=\int_{B} \underline{b} \cdot \underline{\tilde{u}}+\int_{\partial B} \underline{f} \cdot \underline{\tilde{u}} .
$$

We now decompose the forces and the displacements in their respective conjugate quantities, this decomposition is summarized in table 1. With these assumptions the expression of the external work is

$$
L_{e x t}=\int_{S}\left[\underline{b}_{p} \cdot \underline{\tilde{u}}_{0}+b_{w} \tilde{w}_{0}+\underline{b}_{m} \cdot \nabla \tilde{w}_{0}\right]+\int_{\partial B}\left[\underline{f}_{p} \cdot \underline{\tilde{u}}_{0}+f_{w} \tilde{w}_{0}+\underline{f}_{m} \cdot \nabla \tilde{w}_{0}\right] .
$$




\subsubsection{Balance equations}

The principle of virtual work states that

$$
L_{i n t}=L_{e x t} .
$$

Considering equations (8) and (10), the balance equations on $S$ can be obtained, preforming some integrations by parts, as

$$
\begin{aligned}
\nabla \cdot \mathbf{N}+\underline{b}_{p} & =0 \\
\nabla^{2}:(\mathbf{M})+\nabla \cdot\left(\underline{b}_{m}\right)-b_{w} & =0, \\
\dot{q}^{(2)}-\nabla \cdot\left(\underline{J}^{(1)}\right) h_{r} & =0, \\
\dot{q}^{(4)}-\nabla \cdot\left(\underline{J}^{(5)}\right) h_{r} & =0,
\end{aligned}
$$

along with all boundary conditions on $\partial B$ and $\partial(\partial B)$.

\subsection{Constitutive equations}

In this section the constitutive equations of each material used for building the plate are investigated. The aim is to derive the constitutive equations for the plate itself, that is $\mathbf{M}, \mathbf{N}, q^{(l)}$ as functions of the reduced strain parameters and the electric field.

\subsubsection{Elastic plate constitutive equations}

From the linear theory of elasticity we have, for the elastic layer

$$
\mathbf{T}=\mathbb{C} \mathbf{S}
$$

where $\mathbb{C}$ is the fourth order tensor of elasticity.

\subsubsection{Piezoelectric constitutive equations}

According to (IEEE, 1987) the constitutive equations for a piezoelectric material are

$$
\begin{aligned}
\mathbf{T}_{p} & =\mathbb{C}_{p}^{E} \mathbf{S}_{p}-\mathbf{e} \underline{E}, \\
\underline{D} & =\mathbf{e}^{t} \mathbf{S}_{p}+\varepsilon^{S} \underline{E} .
\end{aligned}
$$

Another equivalent form is obtained from the last equations

$$
\begin{array}{r}
\mathbf{S}_{p}=\left(\mathbb{C}_{p}^{E}\right)^{-1} \mathbf{T}_{p}-\mathbf{d} \underline{E}, \\
\underline{D}=\mathbf{d}^{t} \mathbf{S}_{p}+\varepsilon^{T} \underline{E},
\end{array}
$$


where

$$
\mathbf{e}=\mathbb{C}_{p}^{E} \mathbf{d}
$$

We will use these constitutive equations in the hypothesis of thin plates in plane stress and plane strain

\subsubsection{Resistive electrode constitutive equations}

In each resistive layer we have

$$
\underline{J}^{(r)}=\sigma_{r} \underline{E}^{(r)}
$$

the local form of the Ohm Law, in which $\sigma_{r}$ is the specific conductivity $\left[\Omega^{-1} / m\right]$.

For the kinematic assumptions we have

$$
\underline{E}^{(r)}=-\nabla V^{(r)}
$$

and then

$$
\underline{J}^{(r)}=-\sigma \nabla \dot{\psi}^{(r)}=-\frac{1}{\varrho_{r}} \nabla \dot{\psi}^{(r)} .
$$

where $\varrho_{r}$ is the specific resistivity $[\Omega m]$ of the material used for building the electrode.

\subsubsection{PRE plate constitutive equations}

In section 2.3.1 we defined the quantities $\mathbf{N}, \mathbf{M}, q, \underline{J}$. Now that we have introduced constitutive equations for each layer, we can find the PRE plate constitutive equations.

$$
\begin{array}{r}
\mathbf{N}=\mathbb{K}_{N u} \nabla \underline{u}_{0}+k_{N \psi}\left(\dot{\psi}^{(4)}-\dot{\psi}^{(2)}\right), \\
\mathbf{M}=\mathbb{K}_{M w} \nabla^{2} w_{0}+\Gamma\left(\dot{\psi}^{(2)}+\dot{\psi}^{(4)}\right), \\
q^{(2)}=-k_{N \psi} \nabla\left(\underline{u}_{0}\right)-\Gamma \nabla^{2}\left(w_{0}\right)+C_{p} \dot{\psi}^{(2)}, \\
q^{(4)}=k_{N \psi} \nabla\left(\underline{u}_{0}\right)-\Gamma \nabla^{2}\left(w_{0}\right)+C_{p} \dot{\psi}^{(4)}, \\
\underline{J}^{(1)}=-\frac{1}{\varrho_{r}} \nabla \dot{\psi}^{(2)}, \\
\underline{J}^{(5)}=-\frac{1}{\varrho_{r}} \nabla \dot{\psi}^{(4)},
\end{array}
$$


or in compact form

$$
\left[\begin{array}{l}
\mathbf{N} \\
\mathbf{M} \\
q^{(2)} \\
q^{(4)} \\
\underline{J}^{(1)} \\
\underline{J}^{(5)}
\end{array}\right]=\left[\begin{array}{ll:llll}
\mathbb{K}_{N u} & 0 & -k_{N \psi} & k_{N \psi} & 0 & 0 \\
0 & \mathbb{K}_{M w} & \Gamma & \Gamma & 0 & 0 \\
\hdashline-k_{N \psi} & -\Gamma & C_{p} & 0 & 0 & 0 \\
k_{N \psi} & -\Gamma & 0 & C_{p} & 0 & 0 \\
0 & 0 & 0 & 0 & -\frac{1}{\varrho_{r}} & 0 \\
0 & 0 & 0 & 0 & 0 & -\frac{1}{\varrho_{r}}
\end{array}\right]\left[\begin{array}{c}
\nabla\left(\underline{u}_{0}\right) \\
\nabla^{2}\left(w_{0}\right) \\
\dot{\psi}^{(2)} \\
\dot{\psi}^{(4)} \\
\nabla \dot{\psi}^{(2)} \\
\nabla \dot{\psi}^{(4)}
\end{array}\right]
$$

where the coefficients are listed in table 2 and where we introduced $\xi_{p}=h_{p} / h$ as the ratio between the thickness of the piezoelectric layer and that of the elastic layer and

$$
\alpha=8 \xi_{p}^{3}+12 \xi_{p}^{2}+6 \xi_{p}
$$

This parameter $\alpha$ which is related to the ratio between the thickness of the piezoelectric layer and the host plate will also appear in the stiffness terms of the PRE plate equations.

\subsection{Equations of motion}

From equations (12) and using equations (16) we have the complete form of the PRE plate equations

$$
\begin{array}{r}
\mathbb{K}_{N u} \nabla^{2} \underline{u}_{0}+\nabla k_{N \psi}\left(\dot{\psi}^{(2)}-\dot{\psi}^{(4)}\right)+\underline{b}_{p}=0, \\
\mathbb{K}_{M w} \nabla^{4} w_{0}+\Gamma \nabla^{2}\left(\dot{\psi}^{(2)}+\dot{\psi}^{(4)}\right)+\nabla \underline{b}_{m}-b_{w}=0, \\
-k_{N \psi} \nabla\left(\underline{\dot{u}}_{0}\right)-\Gamma \nabla^{2} \dot{w}_{0}+C_{p} \ddot{\psi}^{(2)}-\frac{h_{r}}{\varrho_{r}} \nabla^{2} \dot{\psi}^{(2)}=0, \\
k_{N \psi} \nabla\left(\underline{\dot{u}}_{0}\right)-\Gamma \nabla^{2} \dot{w}_{0}+C_{p} \ddot{\psi}^{(4)}-\frac{h_{r}}{\varrho_{r}} \nabla^{2} \dot{\psi}^{(4)}=0 .
\end{array}
$$

In common applications one can neglect $\underline{b}_{m}$, and then introduce the other forcing terms as

$$
\begin{array}{r}
b_{w}=-\rho_{t} \ddot{w}_{0}+f_{e x t}, \\
\underline{b}_{p}=-\rho_{t} \underline{\ddot{u}}_{0},
\end{array}
$$

where the $\rho_{t}$ is the overall surface mass density, calculated from the volume densities $\rho_{p l}$ of the plate and $\rho_{p z}$ of the piezoelectric material as follows:

$$
\rho_{t}=\rho_{p l} h+\rho_{p z} h_{p} .
$$


Moreover introducing the overall bending stiffness $S_{t}$ as

$$
S_{t}=\frac{h^{3}}{12}\left[\frac{Y}{1-\nu^{2}}+\alpha \frac{Y_{p}}{1-\nu_{p}^{2}}\right],
$$

we have

$$
\begin{array}{r}
\mathbb{K}_{N u} \nabla^{2} \underline{u}_{0}+\rho_{t} \ddot{\underline{u}}_{0}+k_{N \psi} \nabla\left(\dot{\psi}^{(4)}-\dot{\psi}^{(2)}\right)=0, \\
S_{t} \nabla^{4} w_{0}+\rho_{t} \ddot{w}_{0}+\Gamma \nabla^{2}\left(\dot{\psi}^{(4)}+\dot{\psi}^{(2)}\right)=f_{e x t}, \\
C_{p} \ddot{\psi}^{(2)}-\frac{h_{r}}{\varrho_{r}} \nabla^{2} \dot{\psi}^{(2)}-\Gamma \nabla^{2}\left(\dot{w}_{0}\right)-k_{N \psi} \nabla\left(\underline{\dot{u}}_{0}\right)=0, \\
C_{p} \ddot{\psi}^{(4)}-\frac{h_{r}}{\varrho_{r}} \nabla^{2} \dot{\psi}^{(4)}-\Gamma \nabla^{2}\left(\dot{w}_{0}\right)+k_{N \psi} \nabla\left(\underline{\dot{u}}_{0}\right)=0 .
\end{array}
$$

If we consider only bending vibration we have

$$
\nabla^{2} u_{0}+\rho_{p z} \underline{\ddot{u}}_{0}=0
$$

and so

$$
\dot{\psi}^{(4)}=\dot{\psi}^{(2)}=\dot{\psi} .
$$

This derives from the symmetry of the structure with respect to its mean plane, the so called bimorph configuration for the piezoelectric patches, and means that the extensional vibrations are uncoupled from the flux linkage. Then from additional equations, (22c) and (22d), and considering equation (23), the final form for the governing equations in terms of the transverse displacement and of the flux linkage is

$$
\begin{array}{r}
S_{t} \nabla^{4} w_{0}+\rho_{t} \ddot{w}_{0}+2 \Gamma \nabla^{2}(\dot{\psi})=f_{\text {ext }}, \\
C_{p} \ddot{\psi}-\frac{h_{r}}{\varrho_{r}} \nabla^{2} \dot{\psi}-\Gamma \nabla^{2}\left(\dot{w}_{0}\right)=0 .
\end{array}
$$

\section{Dynamical Analysis}

\subsection{Wave propagation in a PRE plate}

In order to investigate the electromechanical behaviour of the PRE plate, it is crucial to study the propagation of electrical and vibrational waves in the structure. This kind of analysis is the best way of determining how the smart structure is reacting to a mechanical input, such as a bending vibration, and how it changes its mechanical properties. To this end we suppose that the plate is infinite and that a bending wave with wave vector $k_{r}$ 
is propagating in the plate. We can then easily decompose the state variables $w_{0}(x, y, t)$ and $\psi(x, y, t)$ as

$$
\left[\begin{array}{c}
w_{0}(x, y, t) \\
\psi(x, y, t)
\end{array}\right]=\left[\begin{array}{l}
W(t) \\
\Psi(t)
\end{array}\right] e^{j \underline{k_{r}} \cdot \underline{r}} .
$$

The system is then reduced as follows

$$
\begin{aligned}
S_{t} k_{r}^{4} W+\rho_{t} \ddot{W}-2 \Gamma k_{r}^{2}(\dot{\Psi}) & =0, \\
C_{p} \ddot{\Psi}+\frac{h_{r}}{\varrho_{r}} k_{r}^{2} \dot{\Psi}+\Gamma k_{r}^{2} \dot{W} & =0,
\end{aligned}
$$

where $k_{r}=\left|\underline{k}_{r}\right|$.

A non-dimensional form of this system, where $\bar{\square}$ is a non dimensional quantity, is

$$
\begin{gathered}
\bar{W}+\ddot{\bar{W}}-2 \gamma \dot{\bar{\Psi}}=0, \\
\ddot{\bar{\Psi}}-\delta \dot{\bar{\Psi}}+\gamma \dot{\bar{W}}=0 .
\end{gathered}
$$

The non dimensional state variables in equations (26) are

$$
\bar{W}=\frac{W}{l_{0}} \quad \bar{\Psi}=\frac{\Psi}{\psi_{0}}
$$

where $\psi_{0}$ and $l_{0}$ are the scaling flux linkage and the scaling displacement, and

$$
\psi_{0}=l_{0} \sqrt{\frac{\rho_{t}}{C_{p}}} .
$$

The non-dimensional electromechanical coupling $\gamma$ and damping coefficient $\delta$ appearing in equations (26) are

$$
\gamma=\frac{2 \Gamma}{\sqrt{C_{p} S_{t}}}, \quad \delta=\frac{h_{r}}{\varrho_{r} C_{p}} \sqrt{\frac{\rho_{t}}{S_{t}}},
$$

From equation (28) we can conclude that $\delta$ is independent of the wavenumber $k_{r}$.

\subsection{Modal model for a simply supported plate}

In this section a modal solution for the PRE plate equations (24) will be developed using Galerkin method. The electromechanical fields $w_{0}(x, y, t)$ and $\psi(x, y, t)$ are given by:

$$
\begin{aligned}
w_{0}(x, y, t) & =\sum_{i=1}^{N} \eta_{i}(t) \phi_{i}(x, y), \\
\psi(x, y, t) & =\sum_{i=1}^{N} \chi_{i}(t) \phi_{i}(x, y),
\end{aligned}
$$


where $\eta_{i}(t)$ and $\chi_{i}(t)$ are Fourier coefficients of the eigenfunctions $\phi_{i}(x, y)$ defined by the eigenvalue problem

$$
\nabla^{4} \phi_{i}=\lambda_{i}^{2} \phi_{i}
$$

along with proper boundary conditions for the displacement field and for the flux linkage. It is important to underline that, in order to keep the electromechanical analogy, the boundary conditions for the electric field must be the analog of the mechanical ones. This implies that the potions of piezoelectric material close to constrained edges of the PEM plate, must be suitably shunted. From equation (30) we obtain the values for natural frequencies

$$
\omega_{i}=\lambda_{i} \sqrt{\frac{S_{t}}{\rho_{t}}} .
$$

Substituting equations (30) and (29) into equations (24), projecting on the given basis a non-dimensional modal form for equations (24), denoting with $\hat{\square}$ a quantity in frequency domain we obtain

$$
\begin{aligned}
\Omega_{i}^{2} \hat{\eta}_{i}-\Omega^{2} \hat{\eta}_{i}-j 2 \gamma \Omega \Omega_{i} \hat{\chi}_{i} & =\hat{f}_{i}, \\
-\Omega^{2} \hat{\chi}_{i}+j \delta \Omega \hat{\chi}_{i}+j \gamma \Omega \Omega_{i} \hat{\eta}_{i} & =0 .
\end{aligned}
$$

where the non-dimensional coupling and damping coefficients are the same as in equations 28 and circular frequencies $\Omega$ and $\Omega_{i}$ are given by

$$
\Omega=\frac{\omega}{\Omega_{0}}, \quad \Omega_{i}=\frac{\omega_{i}}{\Omega_{0}}
$$

where $\Omega_{0}$ is a scaling circular frequency.

The non-dimensional state variables and forcing term in equations (31) are given by

$$
\hat{\eta}=\frac{\eta}{l_{0}}, \quad \hat{\chi}=\frac{\chi}{\psi_{0}}, \quad \hat{f}_{i}=\frac{f_{i}}{F_{0}} .
$$

with the scaling force given by

$$
F_{0}=l_{0} \Omega_{0}^{2} \rho_{t}
$$

From the second equation of system (31) we can obtain

$$
\hat{\chi}_{i}=\frac{j \gamma \Omega \Omega_{i}}{\Omega^{2}-j \delta \Omega \Omega_{i}} \hat{\eta}_{i}
$$


and then, substituting into the first equation of system (31), we have

$$
\Omega_{i}^{2} \hat{\eta}_{i}-\Omega^{2} \hat{\eta}_{i}+\frac{j \gamma \Omega \Omega_{i}}{\Omega^{2}-j \delta \Omega \Omega_{i}} \hat{\eta}_{i}=\hat{f}_{i} .
$$

Finally we have the expression of the Fourier coefficients $\hat{\eta}_{i}$ and $\hat{\chi}_{i}$

$$
\begin{gathered}
\hat{\eta}_{i}=\frac{\hat{f}_{i}}{\Omega_{i}^{2}-\Omega^{2}+\frac{j \gamma \Omega \Omega_{i}}{\Omega^{2}-j \delta \Omega \Omega_{i}}}, \\
\hat{\chi}_{i}=\frac{j \gamma \Omega \Omega_{i} \hat{f}_{i}}{\left(\Omega^{2}-j \delta \Omega \Omega_{i}\right)\left(\Omega_{i}^{2}-\Omega^{2}+\frac{j \gamma \Omega \Omega_{i}}{\Omega^{2}-j \delta \Omega \Omega_{i}}\right)} .
\end{gathered}
$$

\subsection{Optimization}

When studying the behaviour of the system it is necessary to analyse both the transient response and the forced response. In order to do this we will introduce two different quantities: the decay rate $D(\delta, \gamma)$ for the transient response and the mobility function $H(\hat{\omega}, \delta, \gamma)$ for the forced response, defined as

$$
\begin{gathered}
D(\delta)=\min _{i=1,2,3}\left(\left|\operatorname{Re}\left(\lambda_{i}\right)\right|\right), \\
H(\Omega, \delta, \gamma)=\frac{j \Omega \hat{\eta}_{i}(\Omega, \delta, \gamma)}{\hat{f}_{i}} .
\end{gathered}
$$

The parameter that will be optimized is the damping $\delta$ and the resulting value $\delta_{o p t}$ will be used in equation (28) to determine the optimal value for the specific resistivity for a given thickness of the electrode.The piezoelectric layer thickness has been optimized maximizing the coupling coefficient $\gamma$ with a total added mass constraint of $40 \%$.

\subsubsection{Transient response}

In order to maximize the damping efficiency, a pole placement criterion will be used for finding the optimal value for $\delta_{\text {opt }}$ that maximizes $D(\delta)$. The system (26) is homogeneous, thus it admits exponential solutions

$$
\left[\begin{array}{l}
\bar{W} \\
\bar{\Psi}
\end{array}\right]=\left[\begin{array}{l}
\overline{\bar{W}} \\
\overline{\bar{\Psi}}
\end{array}\right] e^{\lambda t} .
$$

The Pole Placement criterion consists in finding the roots $\lambda$ of system (26) imposing the wave solution (36) and then finding the value of $\delta$ witch maximizes the decay rate. This method gives the following results: 


$$
\delta_{\text {opt }}^{P P}=1+\frac{\gamma^{2}}{2},
$$

for which it is

$$
D_{o p t}=\frac{\gamma^{2}}{4}
$$

Since $\delta$ is independent of the wavenumber $k_{r}$, the optimal damping condition holds for each wavenumber, and leads to the optimal value for the resistance

$$
\varrho_{o p t}=\frac{h_{r}}{C_{p}} \sqrt{\frac{\rho_{t}}{S_{t}}}
$$

\subsubsection{Forced response}

Using the results obtained in section (3.2) it is easy to determine the mobility function , defined as

$$
H(\Omega, \delta, \gamma)=\frac{j \Omega}{\Omega_{i}^{2}-\Omega^{2}+\frac{j \gamma \Omega \Omega_{i}}{\Omega^{2}-j \delta \Omega \Omega_{i}}},
$$

For optimizing the forced response a widely adopted approach consists in minimizing the $\infty-$ norm of the mobility function, defined as

$$
\|H(\Omega, \delta, \gamma)\|_{\infty}=\sup _{\Omega \in R}|H(\Omega, \delta, \gamma)| .
$$

It is known in the literature, see (Den Hartog, 1956), that in coupled systems there exists a so-called fixed point $F=\left(\Omega_{F},\left|H\left(\Omega_{F}, \delta, \gamma\right)\right|\right)$ defined as follows

$$
\forall \bar{\gamma} \in R^{+} \quad \exists \Omega_{F} / \forall \delta_{1}, \delta_{2} \in R^{+}\left|H\left(\Omega_{F}, \delta_{1}, \bar{\gamma}\right)\right|=\left|H\left(\Omega_{F}, \delta_{2}, \bar{\gamma}\right)\right| .
$$

This point corresponds to the circular frequency

$$
\Omega_{F}(\gamma)=\sqrt{1+\frac{\gamma^{2}}{2}}
$$

Imposing that the maximum of $|H(\Omega, \delta, \gamma)|$ is at $\Omega_{F}$ we find the optimal value for $\delta$ :

$$
\frac{\partial}{\partial \Omega}\left|H\left(\Omega_{F}, \delta, \gamma\right)\right|_{\Omega=\Omega_{F}}=0 \Rightarrow \delta_{o p t}^{F P}=\sqrt{\frac{8+10 \gamma^{2}+3 \gamma^{3}}{8+2 \gamma^{2}}} \simeq 1+\frac{\gamma^{2}}{2} .
$$

The last approximation holds because $\gamma$ is small, in practical situations it is

$$
0<\gamma<0.3
$$

As expected, $\delta_{o p t}^{P P} \simeq \delta_{o p t}^{F P}$ so the two criteria give the same result. 


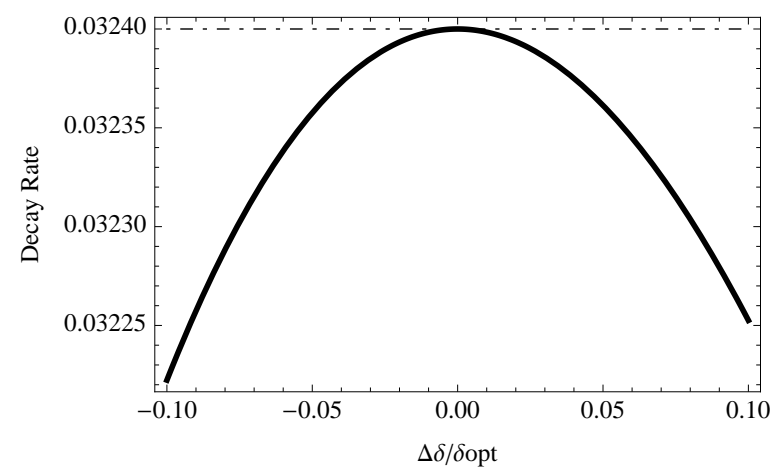

(a) Decay Rate

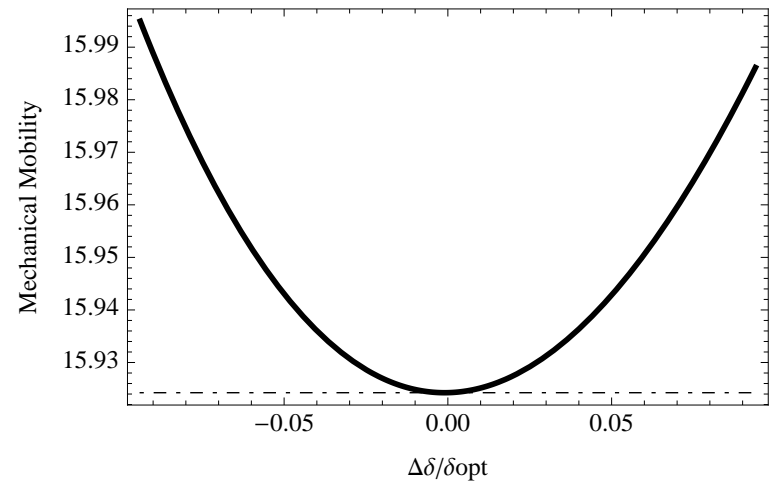

(b) Mechanical Mobility $\|H\|_{\infty}$

Figure 4: Plot of the performance indexes of the two optimization methods as a function of damping $\delta$.

\subsubsection{Effect of parameter variations}

The plots in figure 4 show the decay rate and mechanical mobility as functions of the percentage variations of the parameter $\delta$ with respect to the optimum value $\delta_{\text {opt }}$. Both plots show that relatively high variations of $\delta$ near the optimum value do not affect very much the overall behaviour and the damping performances of the structure. This because, as shown in the plots, the derivatives of both curves calculated for $\Delta \delta / \delta_{\text {opt }}$ are horizontal.

\section{Acoustic Analysis}

\subsection{Acoustic performance indices}

Let us consider an Euclidean space with its basis $\left(\underline{e}_{1}, \underline{e}_{2}, \underline{e}_{3}\right)$. We denote a generic point of the plate by $\underline{r}=x \underline{e}_{1}+y \underline{e}_{2}$ and a generic point in the space as $\underline{p}=x \underline{e}_{1}+y \underline{e}_{2}+z \underline{e}_{3}$. 
The plate separates two identical light fluid media with density $\rho$.

Far Field Pressure. The pressure $\widehat{p}(\underline{p}, \omega)$ in the generic point of the space due to the motion of a portion $\mathrm{S}$ of the plate can be calculated using the Rayleigh formula:

$$
\widehat{p}(\underline{p}, \omega, S)=\frac{j \omega \rho}{2 \pi} \int_{S} \frac{e^{-j k(\underline{p}-\underline{r})}}{\underline{p}-\underline{r}} \widehat{w}_{0}(\underline{r}, \omega) d S,
$$

as explained in (Cremer, 1973; Fahy, 2001; Filippi, 1984).

This is a very important performance index, since it is related to a physical quantity that can be easily measured, such as the pressure. In the literature there exists a standard method for measuring this quantity, see (ISO 3745, 1977).

Radiated power at the plate level. The radiated power $\pi_{r}$ at the plate level can be expressed by the surface integral of the product between the pressure in each point of the plate, calculated with equation (39), multiplied by its velocity:

$$
\pi_{r}(\omega, S)=\frac{1}{2} \int_{S} \operatorname{Re}\left\{\left(\left.\hat{p}(\underline{r}, \omega)\right|_{z=0}\right) \cdot\left(j \omega \hat{w}_{0}(\underline{r}, \omega)\right)^{*}\right\} d S,
$$

where $\square^{*}$ is a conjugate quantity.

This is a global parameter that gives us different informations with respect to the far field pressure, since it involves also the near field, but it is not a measurable quantity.

Sound Reduction Index. Sound transmission through a panel is usually studied in the literature by considering an infinite plate, see (Cremer, 1973; Fahy, 2001; Filippi, 1984). This approximation is based on the fact that for higher frequencies the wavelength of the bending vibrations is much smaller than the characteristic dimension of the structure. Then we consider two semi-infinite sound media separated by an infinite plate placed at $z=0$.

The wave propagating in the two media must satisfy the wave equation

$$
\nabla^{2} \hat{p}_{n}(\underline{r}, z, \omega)+\frac{1}{c^{2}} \hat{p}_{n}(\underline{r}, z, \omega)=0, \quad n=1,2,
$$

where $c$ is the speed of sound in the medium.

We now introduce the incident power on a surface $S$ as

$$
\pi_{i}(\theta, \omega, \phi, S)=\frac{1}{2} \int_{S} \operatorname{Re}\left\{\left(\left.\hat{p}_{1}(\underline{p}, \omega)\right|_{z=0}\right) \cdot\left(j \omega \hat{w}_{0}(\underline{r}, \omega)\right)^{*}\right\} d S,
$$


where $\theta$ and $\phi$ the angles that the wave vector of the incident wave forms with $\underline{e}_{1}$ and $\underline{e}_{2}$, respectively; for the sake of simplicity we will consider $\phi=0$ without loss of generality. We can define, using equation (40), the transparence function by

$$
\tau(\theta, \omega)=\frac{\pi_{t}}{\pi_{i}} .
$$

This function represents the fraction of the incident power that actually passes through the plate. When testing the acoustical behaviour of a structure, a standard parameter used is the Sound Reduction Index:

$$
S R I(\theta, \omega)=10 \log \left(\frac{1}{\tau(\theta, \omega)}\right) .
$$

\subsection{Simulations}

In this section some numerical simulations are considered, in order to show the performances of the control system, compared with two other passive control strategies: a plate with an attached viscoelastic layer and a Piezo Electro Mechanical (PEM) plate. The viscoelastic plate is composed by an aluminium plate with an attached viscoelastic layer. Following (Cremer, 1973), the thickness of the viscoelastic layer has been chosen equal to $h$. The material used in simulations is a 3M ISD 112. This structure represents the most simple way to introduce frequency independent damping.

The PEM plate is a smart structure composed by an aluminium plate with a distributed matrix of piezoelectric transducers, interconnected by a passive modular network of inductors and resistors witch assures a frequency independent damping. An accurate description of the system is in Alessandroni et al. (2004). This structure uses the equivalent amount of piezoelectric material as a PRE plate. It is important to denote that, being the PEM plate a discrete structure, the size of each transducer must be smaller than the considered wavelengths. Consequently the number of connections needed increases with the maximum frequency of interest, that is the main trade off of this control technique, along with the problems related to mistuning.

All the parameters used for the simulation presented in this section are listed in the Appendix.

Far Field Pressure. According to (ISO 3745, 1977) the far field sound pressure can be estimated using the pressure calculated in 10 points optimally placed in an hemisphere. 
We can use equation (39) to determine the sound pressure on these points, and then we consider the mean value in order to have a global parameter for the far field sound radiation. A numerical method is proposed in (Bai and Tsao, 2002) and will be applied to calculate the sound pressure. In figure 5 (c) the mean pressure field radiated by a PRE plate is compared with those of an aluminium plate without control, a viscoelastic plate and a PEM plate. The PRE plate provides a considerable reduction of the mean value of the far field pressure near the peaks introduced by the most radiating structural modes and introduces more damping than a viscoelastic plate. The PEM plate is more efficient, and this was to be expected since it introduces electrical resonances for each mode maximizing the dissipated energy.

Radiated power at the plate level. This analysis is performed on a finite simply supported plate. The acoustic power $W$ on the surface of the plate can be expressed equation (40). Since from the modal model, section 3.2, we know the distribution of the velocity field in each point of the structure we can use equation (39) to obtain an expression of the radiated power which depends only on that known velocity field.

This integral will be calculated in a numerical way, using the method introduced in (Elliott and Johnson, 1993) and then slightly modified in (Bai and Tsao, 2002) with the results from (Borgiotti and Jones, 1994). The idea is to divide the structure into a matrix of $M=n \times n$ elementary elements, radiating as pistons, in order to extract from the geometry of the structure some of its acoustic properties. In figure 5(b) the radiated sound power plot for a finite simply supported plate is shown. Also with this performance parameter we can see how the control system is optimized for each radiating structural modes and the results are in line with those obtained for the far field sound pressure.

Sound Reduction Index. For finding the SRI we need the solution of equations (41) together with equations (24) when considering $f_{e x t}=p_{1}-p_{2}$ and the following continuity conditions

$$
\left.\frac{\partial p_{n}}{\partial z}\right|_{z=0}=-\rho \ddot{w}_{0}, \quad n=1,2 .
$$


The scaling quantities are listed in the Appendix. As discussed in section 3.1 we can use the wave equation as solution of this coupled system, in the form

$$
\left[\begin{array}{c}
\hat{w}_{0}(\underline{r}, \Omega) \\
\hat{\psi}(\underline{r}, \Omega) \\
\hat{p}_{1}(\underline{r}, z, \Omega) \\
\hat{p}_{2}(\underline{r}, z, \Omega)
\end{array}\right]=\left[\begin{array}{ccc}
\hat{W}(\Omega) & 0 & 0 \\
\hat{\Psi}(\Omega) & 0 & 0 \\
0 & \hat{P}_{i}(\Omega) & \hat{P}_{r}(\Omega) \\
0 & \hat{P}_{t}(\Omega) & 0
\end{array}\right]\left[\begin{array}{c}
1 \\
e^{-j \hat{k}_{z} \hat{z}} \\
e^{j \hat{k}_{z} \hat{z}}
\end{array}\right] e^{j \hat{k}_{r} \cdot \hat{\underline{r}}},
$$

where

$$
\hat{k}_{z}=\Omega \cos \theta, \quad\left|\underline{\hat{k}_{r}}\right|=\hat{k}_{r}=\Omega \sin \theta,
$$

and where $\theta$ is the angle of the incident wave.

Considering the solution (46), considering $f_{e x t}=p_{1}-p_{2}$ and equation (41), system (24) becomes

$$
\begin{array}{r}
\hat{k}_{r}^{4} \hat{W}-\Omega^{2} \hat{W}-2 \gamma j \Omega \hat{k}_{r}^{2} \hat{\Psi}=\hat{P}_{i}+\hat{P}_{r}+\hat{P}_{t} \\
-\Omega^{2} \hat{\Psi}+\delta j \Omega \hat{k}_{r}^{2} \hat{\Psi}+\gamma j \Omega \hat{k}_{r}^{2} \hat{W}=0 \\
j \hat{k}_{z}\left(\hat{P}_{r}+\hat{P}_{r}\right)=\zeta \Omega^{2} \hat{W} \\
j \hat{k}_{z} \hat{P}_{t}=\zeta \Omega^{2} \hat{W}
\end{array}
$$

From equations (42), (43), (44), (48), and considering conditions (47), we have

$$
S R I(\theta, \Omega)=10 \log \left(\frac{\left|\hat{k}_{r}^{4}-\Omega^{2}+\frac{2 \gamma^{2} \Omega^{2} \hat{k}_{r}^{4}}{j \delta \Omega \hat{k}_{r}^{2}-\Omega^{2}}+\frac{2 \zeta \Omega^{2}}{j k_{z}}\right|^{2}}{\Omega^{2} \frac{4 \zeta}{\cos \theta}}\right)
$$

. In figure 5(a) the Sound Reduction Index for an aluminium plate, a PRE plate, a viscoelastic plate and a PEM plate are shown. Since all considered control systems control system are based on damping, no enhancement is possible in the region controlled by mass, below the critical frequency $f_{c r}$, where for the selected structure $f_{c r}=5600 \mathrm{~Hz}$. A detailed definition of $f_{c r}$ is in (Filippi, 1984). Above the critical frequency, since both PRE and viscoelastic controls are frequency independent, there is a linear enhancement and the performances of the PRE plate are better than both those of a standard viscoelastic plate and of a PEM plate. 


\subsection{Effect of parameter variations}

As a consequence of the results obtained in section 3.3, small variations of the damping parameter $\delta$ do not affect very much the quality of the control. In figure 6 we have plotted the effect of the variation of $\delta$ on the three performance indices.

Hence when considering industrial applications, the incertitudes in the thickness of the resistive layer $h_{r}$ or of its resistivity $\varrho_{r}$ introduced by the industrial process, will not compromise the behaviour of the PRE plate. On the other hand, when considering control strategy based on resonant effects, such as those presented in (Carneal and Fuller, 2004; Behrens et al., 2003; Lee and Chen, 1999b; Zhang et al., 2004; Ozer and Royston, 2003; Batra et al., 2005) as well as in (Maurini, 2005; dell'Isola et al., 2003; Alessandroni et al., 2004, 2002), the sensitivity with respect to the tuning parameter is very high, and this is one of the main problems when considering industrial applications of these kind of structures.

As an example the effect of small variations of the tuning parameter $\beta$ on the performances of a PEM plate on a target mode and on the sound reduction index are plotted in figure 7 .

\section{Conclusions}

As shown in section 4.2 the proposed smart structure contributes to a considerable improvement of the acoustic isolation properties of a thin plate, with respect to the acoustic performance index considered, i.e. far field sound pressure, radiated sound power at plate level and Sound Reduction Index.

A comparison with a standard viscoelastic damping approach and with another passive piezoelectric structure that introduces frequency independent control, the PEM plate, shows how the PRE plate performs a trade off between the two structures (figure 5). The PRE plate results more efficient in sound radiation and transmission control than a viscoelastic plate, and it is also important to denote that the piezoelectric layer is thinner than the viscoelastic one, and this can be an advantage in terms of weight optimization for high level applications.

The better behaviour of the PEM plate was to be expected, being the PEM plate a resonant circuit. Despite this fact, the advantages of using a PRE plates are clear when 
considering the effects of parameters variations and when comparing the complexity of the required circuitry. A detailed study of the dynamic and acoustic behaviour of the proposed smart plate structure shows how the control is frequency independent and not very sensitive of parameter variations. The same analysis performed for the PEM plate shows how sensible a resonant structure is to the tuning parameter variation.

Moreover it is crucial to underline that the PRE plate contains itself a real embedded circuit, and that does not need any external power supply and any connection with external circuitry. Other passive smart structures, such as the PEM plate or other RL shunt based controllers, need very complex external circuitry that introduce additional weight and required maintenance. This consideration, along with the distributed nature of the circuit means that, with a particular industrial process, it is possible to build smart panels that only need to be assembled, since the optimal parameters of the circuit are determined at construction level.

The drawback of this structure in terms of realizability consists in finding an industrial process for building the piezoelectric layer, that is much bigger than the commercial components available. This problem can be avoided by using multiple patches immersed in an unique resistive material, or using piezoelectric fibers.

Further applications and extensions should concentrate on the construction of a prototype and on the addition of mechanical properties, e.g. viscoelastic behaviour, to the resistive layer.

\section{Acknowledgements}

The authors would like to thank Corrado Maurini (Institut Jean Le Rond d'Alembert, Université Pierre et Marie Curie, Paris 6) for the support in conceiving this article, and the "Tullio Levi Civita" Foundation for the financial support.

\section{Appendix}

All plots presented in previous sections have been realized considering a rectangular plate made of aluminium having the characteristics presented in Table 3.

The plate is assumed to be covered by a piezoelectric layer having the properties shown in Table 5 . 
Table 1: Decomposition of external forces with respect to the conjugate virtual quantities.

\begin{tabular}{lll}
\hline & Force & Conjugate \\
\hline \multirow{3}{*}{ Body } & $\underline{b}_{p}=\sum_{i} \int_{I_{i}} \underline{b}$ & $\underline{\tilde{u}}_{0}$ \\
& $b_{w}=\sum_{i} \int_{I_{i}} \underline{b} \cdot \underline{e}_{3}$ & $\tilde{w}_{0}$ \\
$\underline{b}_{m}$ & $=-\sum_{i} \int_{I_{i}} z \underline{b}$ & $\nabla \tilde{w}_{0}$ \\
\hline \multirow{3}{*}{ Surface } & $\underline{f}_{p}=\sum_{i} \int_{I_{i}} \underline{f}$ & $\underline{\tilde{u}}_{0}$ \\
& $\underline{f}_{w} \int_{I_{i}} \underline{f} \cdot \underline{e}_{3}$ & $\tilde{w}_{0}$ \\
$\underline{f}_{m}$ & $=\sum_{i} \int_{I_{i}} z \underline{f}$ & $\nabla \tilde{w}_{0}$ \\
\hline
\end{tabular}

Table 2: Coefficients in Constitutive Equations

\begin{tabular}{lll}
\hline Coefficient & Value & Description \\
\hline $\mathbb{K}_{M w}$ & $\frac{h^{3}}{12}\left[\mathbb{C}^{E}+\alpha \mathbb{C}_{p}^{E}\right]$ & Bending Stiffness \\
$\Gamma$ & $\frac{d_{31} Y_{p z t} h\left(\xi_{p}+1\right)}{2\left(1-\nu_{p}\right)}$ & Bending Coupling \\
$\mathbb{K}_{N u}$ & $h\left(\mathbb{C}^{E}+2 \xi_{p} \mathbb{C}_{p}^{E}\right)$ & Extensional Stiffness \\
$k_{N \psi}$ & $\frac{d_{31} Y}{(1-\nu)}$ & Extensional Coupling \\
$C_{p}$ & $\frac{\varepsilon_{33}^{S}}{h_{p}}$ & Capacitance \\
\hline
\end{tabular}

Table 3: Aluminium plate constitutive parameters and dimensions

\begin{tabular}{lll}
\hline Coefficient & Value & Description \\
\hline$Y$ & $70 \times 10^{9} \mathrm{~N} / \mathrm{m}^{2}$ & Young Modulus \\
$\nu$ & .33 & Poisson Ratio \\
$\rho$ & $2700 \mathrm{Kg} / \mathrm{m}^{3}$ & Mass density \\
$a$ & $.21 \mathrm{~m}$ & Length \\
$b$ & $.30 \mathrm{~m}$ & Width \\
$h$ & $2 \times 10^{-3} \mathrm{~m}$ & Thickness \\
\hline
\end{tabular}

Table 4: Viscoelastic material parameters and dimensions

\begin{tabular}{lll}
\hline Coefficient & Value & Description \\
\hline$Y_{v}^{*}$ & See reference 3M (1993) & Complex Young Modulus \\
$\nu_{v}$ & .49 & Poisson Ratio \\
$\rho_{v}$ & $1000 \mathrm{Kg} / \mathrm{m}^{3}$ & Mass density \\
$h_{v}$ & $2 \times 10^{-3} \mathrm{~m}$ & Thickness \\
\hline
\end{tabular}

The properties of the viscoelastic layer are listed in Table 4.

The characteristic quantities in the simulations are presented in Table 6 .

The optimal values for the resistive layer are presented in Table 7 . 
Table 5: Piezoelectric transducers constitutive parameters and dimensions

\begin{tabular}{lll}
\hline Coefficient & Value & Description \\
\hline$Y_{p}$ & $6.6 \times 10^{10} \mathrm{~N} / \mathrm{m}^{2}$ & Young Modulus \\
$\nu_{p}$ & .29 & Poisson Ratio \\
$\rho_{p}$ & $7500 \mathrm{Kg} / \mathrm{m}^{3}$ & Mass density \\
$d_{31}$ & $-274 \times 10^{-12} \mathrm{~m} / \mathrm{V}$ & Coupling coefficient \\
$\varepsilon_{33}^{S}$ & $3.0104 \times 10^{-8} \mathrm{Fm}-1$ & Dielectric constant \\
$h_{p}$ & $0.267 \times 10^{-3} \mathrm{~m}$ & Thickness \\
\hline
\end{tabular}

Table 6: Characteristic quantities

\begin{tabular}{lll}
\hline Coefficient & Value & Description \\
\hline$t_{0}$ & $1 \mathrm{~s}$ & Characteristic time \\
$\Psi_{0}$ & $1 \mathrm{Vs}$ & Characteristic flux linkage \\
$W_{0}$ & $10 \times 10^{-3} \mathrm{~m}$ & Characteristic deflection \\
\hline
\end{tabular}

Table 7: Resistive layer properties

\begin{tabular}{lll}
\hline Coefficient & Value & Description \\
\hline$h_{r}$ & $1 \times 10^{-4} m$ & Thickness \\
$\varrho_{r}$ & $.231 \Omega \cdot m$ & Specific resistivity \\
\hline
\end{tabular}

\section{References}

3M. ScotchDampTM vibration control systems. (MN USA): 3M Specialties Division, 1993.

Alessandroni, S., Andreaus, U., dell'Isola, F., Porfiri, M., 2004. Piezo-electromechanical (pem) kirchhofflove plates. European Journal of Mechanics A/Solids 23, 689-702.

Alessandroni, S., dell'Isola, F., Porfiri, M., 2002. A revival of electric analogs for vibrating mechanical systems aimed to their efficient control by pzt actuators. International Journal of Solids and Structures 39 (5295-5324).

Bai, M. R., Tsao, M., 2002. Estimation of sound power of baffled planar sources using radiation matrices. Journal of the Acoustical Society of America 112 (3), 876-883.

Batra, R., dell'Isola, F., Vidoli, S., Vigilante, D., 2005. Multimode vibration suppression with passive two-terminal distributed network incorporating piezoceramic transducers. International Journal of Solids and Structures 42, 3115-3132.

Behrens, S., Moheimani, S., Fleming, A., 2003. Multiple mode current flowing passive piezoelectric shunt controller. Journal of Sound and Vibration 266, 929-942.

Borgiotti, G. V., Jones, K. E., 1994. Frequency independence property of radiation spatial filters. Journal of the Acoustical Society of America 96 (6), 3516-3524.

Carneal, J. P., Fuller, C., 2004. An analytical and experimental investigation of active structural acoustic control of noise transmission through double panel systems. Journal of Sound and Vibration 272, 749771 .

Cremer, Heckl, U., 1973. Structure Borne Sound. Springer-Verlag.

dell'Isola, F., Porfiri, M., Vidoli, S., 2003. Piezo-electromechanical (pem) structures: passive vibration control using distributed piezoelectric transducers. Comptes Rendus de l'Academie des Sciences, Mécanique 331, 69-76.

Den Hartog, J., 1956. Mechanical vibrations. New York, McGraw-Hill.

Elliott, S. J., Johnson, M. E., October 1993. Radiation modes and the active control of sound power. Journal of Acoustical Society of America 94 (4), 1993.

Fahy, F., 2001. Fundations of Acoustical Engineering. Elsevier.

Filippi, P. J. T., 1984. Acoustique Générale. Les editions de phisique.

Gardonio, P., Bianchi, E., Elliott, S., 2004. Smart panel with multiple decentralized units for the control of sound transmission. part i: theoretical predictions. Journal of Sound and Vibration 274, $163-192$. 
Gardonio, P., Elliott, S. J., April 2005. Smart panels with velocity feedback control systems using triangularly shaped strain actuators. Journal of the Acoustical Society of America 117 (4), $2046-$ 2064.

Halim, D., Moheimani, S. R., 2003. An optimization approach to optimal placement of collocated piezoelectric actuators and sensors on a thin plate. Mechatronics 13, 27-47.

IEEE, 1987. IEEE Standard on Piezoelectricity - IEEE Std 176-1987. Institute of Electrical and Electronic Engineers.

ISO 3745, 1977. ISO 3745, Acoustics-Determination of sound power levels of noise sources-Precision methods for anechoic and semi-anechoic rooms, 1977. ISO 3745, Acoustics-Determination of sound power levels of noise sources-Precision methods for anechoic and semi-anechoic rooms.

Lee, J., Chen, J., 1999a. Active control of sound radiation from a rectangular plate excited by line moment. Journal of Sound and Vibration 220 (1), 99-115.

Lee, J. C., Chen, J. C., 1999b. Active control of sound radiation from rectangular plates using multiple piezoelectric actuators. Applied Acoustics 57, 327-343.

Maurini, C., 2005. Piezoelectric composites for distributed passive electric control: beam modelling, modal analysis, and experimental implementation. Ph.D. thesis, Université Paris 6 - Sapienza Università di Roma.

Maurini, C., dell'Isola, F., Del Vescovo, D., SEP 2004. Comparison of piezoelectronic networks acting as distributed vibration absorbers. mechanical system and signal processing 18 (5), 1243-1271.

Ozer, M. B., Royston, T. J., 2003. Passively minimizing structural sound radiation using shunted piezoelectric materials. Journal of Acoustical Society of America 114 (4), 1934-1946.

Strassberger, M., Waller, H., 2000. Active noise reduction by structural control using piezoelectric actuators. Mechatronics 10, 851-868.

Wallace, C., 1972. Radiation resistance of a rectangular panel. Journal of the Acoustical Society of America 51 (3), 946-952.

Zhang, W., Qiu, J., Tani, J., 2004. Robust vibration control of a plate using self-sensing actuators of piezoelectric patches. Journal of Intelligent Material Systems and Structures 15, 923-931. 


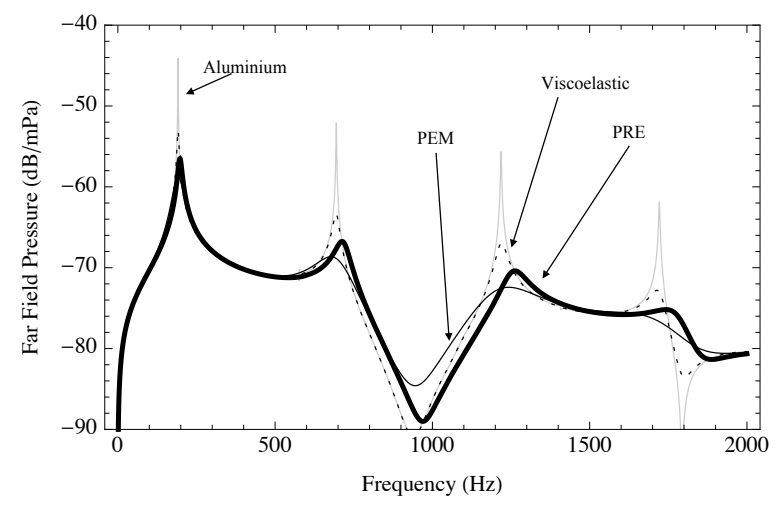

(a) Far Field Pressure

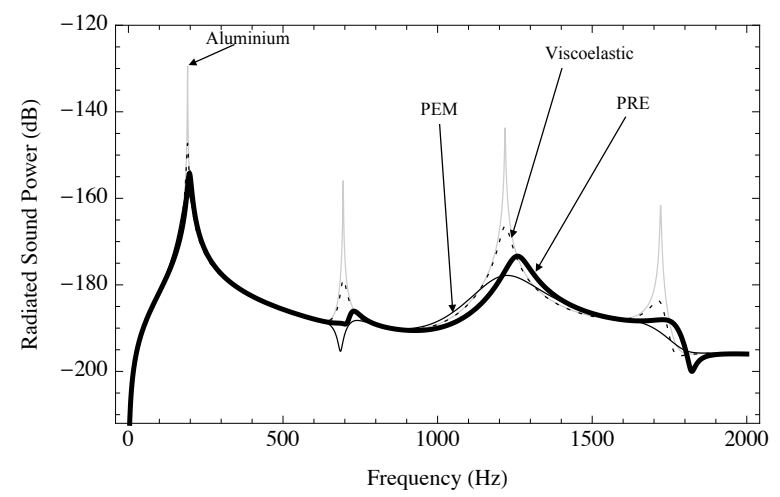

(b) Radiated Sound Power

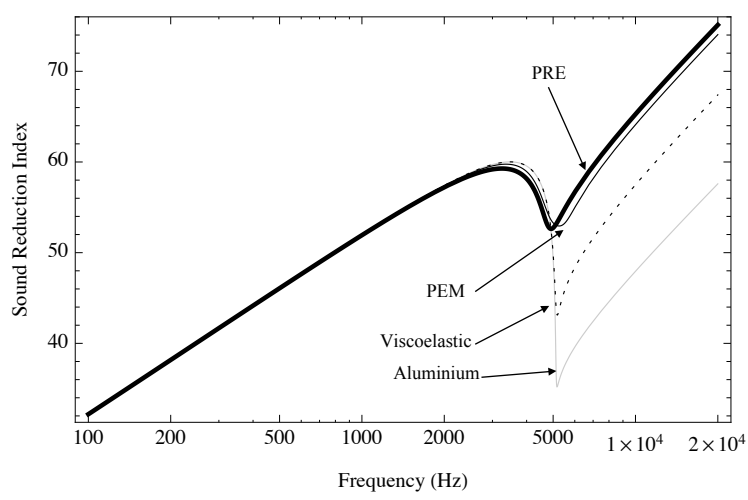

(c) Sound Reduction Index

Figure 5: Sound Radiation Index, Radiated Sound Power and Far Field Pressure of an Aluminium plate, a PRE Plate, a PEM plate and a plate with viscoelastic treatment. 


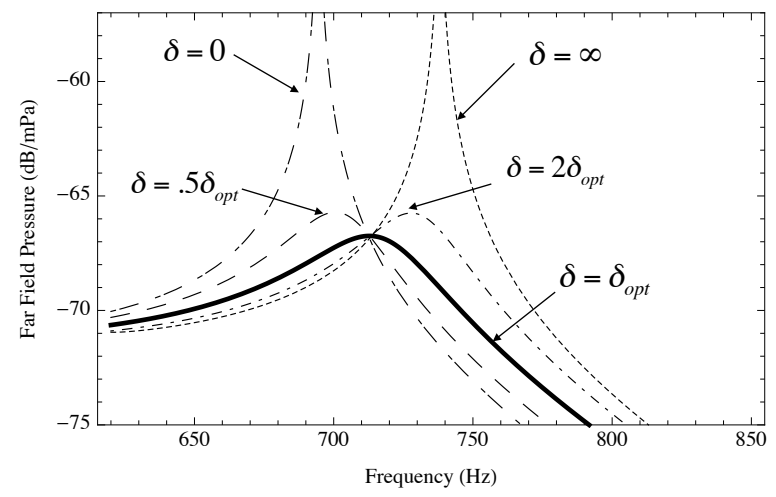

(a) Far Field Pressure

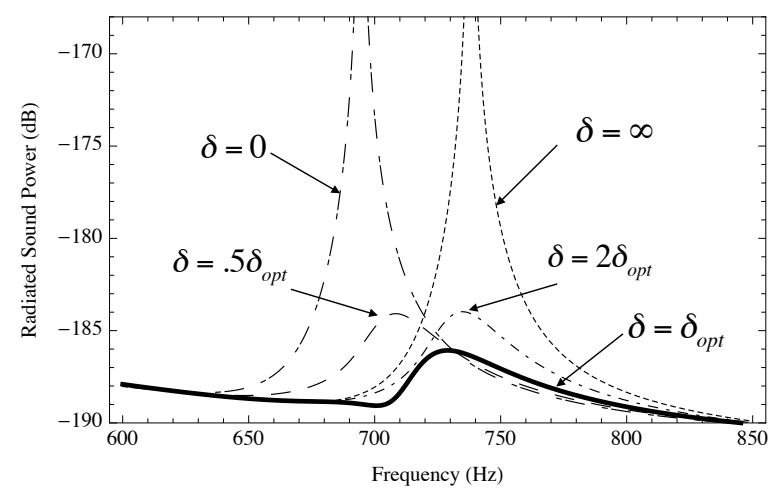

(b) Radiated Sound Power

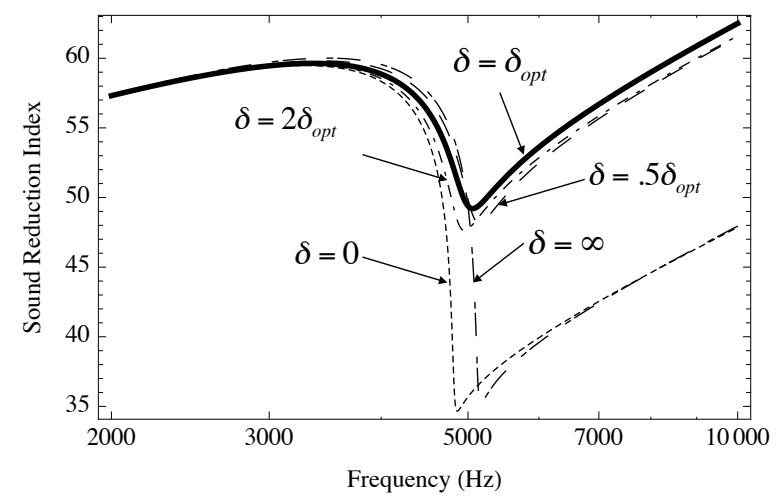

(c) Sound Reduction Index

Figure 6: Sound Radiation Index, Radiated Sound Power and Far Field Pressure for a PRE plate as a function of $\delta$. 


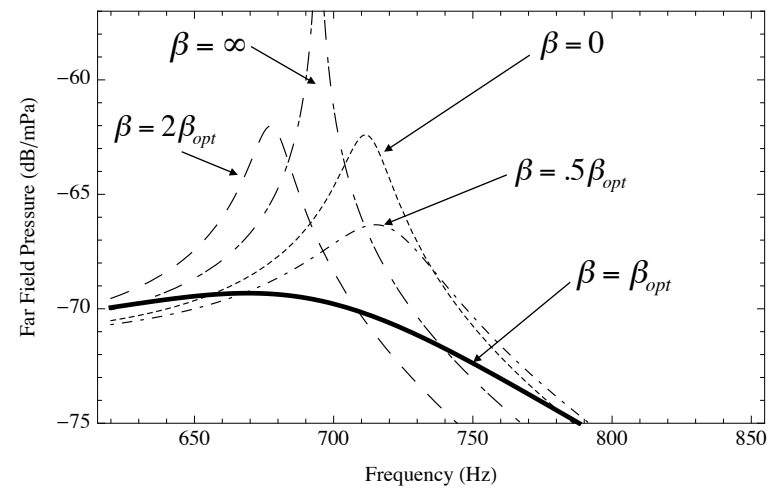

(a) Far Field Pressure

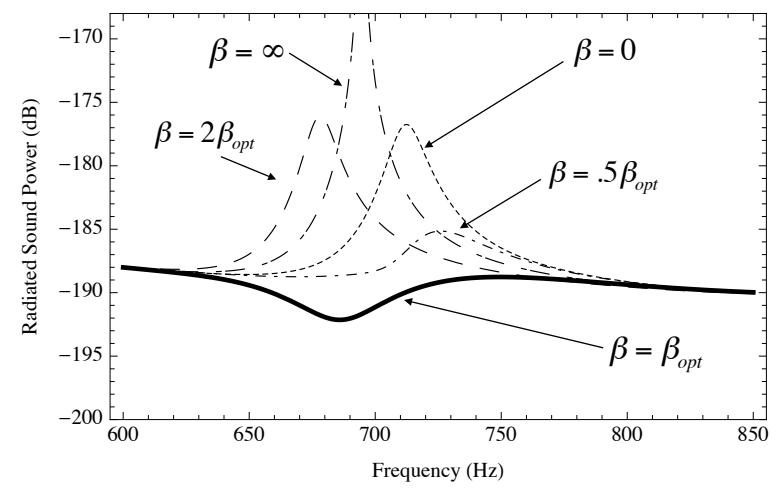

(b) Radiated Sound Power

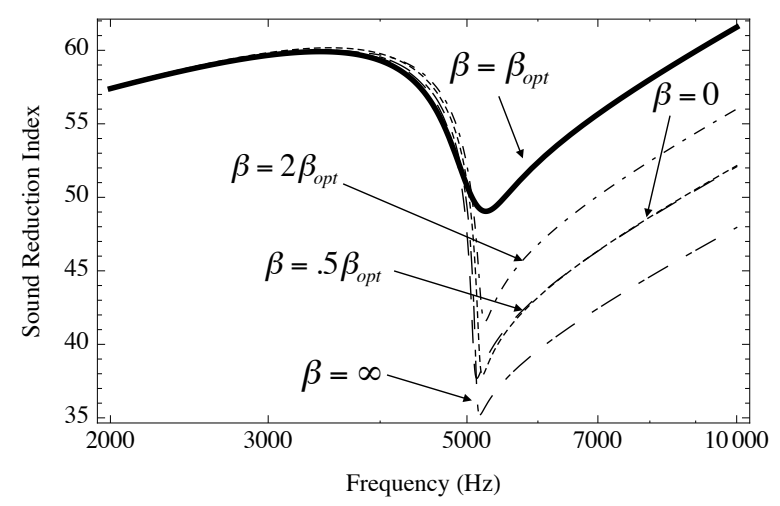

(c) Sound Reduction Index

Figure 7: Sound Radiation Index, Radiated Sound Power and Far Field Pressure for a PEM plate as a function of the tuning parameter. 